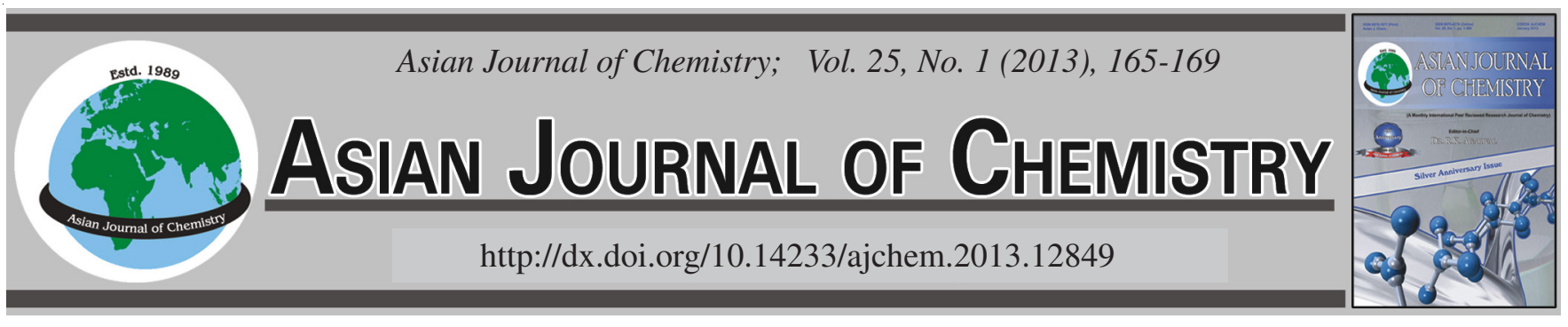

\title{
Response of Anopheles stephensi to Deltamethrin in Lahore District, Pakistan
}

\author{
ShafaAt Yar Khan ${ }^{1}$, Abida Butt ${ }^{2}$, Hafiz Muhammad Tahir ${ }^{2, *}$ and Shazia ShafaAt ${ }^{3}$
}

\begin{abstract}
${ }^{1}$ Department of Biological Sciences, University of Sarghoda, Sarghoda, Pakistan
${ }^{2}$ Department of Zoology, University of the Punjab, Lahore, Pakistan

${ }^{3}$ Department of Zoology, Government College University, Lahore, Pakistan
\end{abstract}

*Corresponding author: E-mail: hafiztahirpk1@yahoo.com

\begin{abstract}
In the present study, both males and females of Anopheles stephensi Liston were sampled from three different localities of Lahore district of Punjab, Pakistan. (Jaman, Mohlanwal and Noorpur Bhatta A1). Bioassays against $0.05 \%$ deltamethrin were performed to evaluate the level of resistance in An. stephansi. All three populations were found susceptible to the WHO recommended dose of deltamethrin. Mortality rates were significantly higher in males than females. Biochemical tests were performed to measure the esterase activity, both in males and females of An. stephensi. All three populations displayed a large variation in the levels of both estrases A and B. Females were found to have significantly higher activity of esterase A and esterase B than males. Only one band (A1) was observed for esterase A in all three populations. For esterase B two bands (B1 and B3) were observed in Mohlanwal and Noorpur Bhatta A1. However, in Jaman a third bands (B2) of moderate intensity was also present between B1 and B3. Results of the present study suggested that present variations and high level of esterases in populations of An. stephensi in district Lahore are not associated with resistance against deltamethrin.
\end{abstract}

Key Words: Esterases, Anopheles stephensi, Resistance, Pyrethroids.

\section{INTRODUCTION}

Mosquitoes of genus Anopheles not only cause a severe biting nuisance, primarily in the summer months in Pakistan but also are a serious threat to public health. There are over 577 species of this genus throughout the world out of which 77 species are confirmed vectors for malaria. In Pakistan about 24 species of Anopheles are reported and among them two are major malarial vector species An. culicifacies Giles and $A n$. stephensi. Two new species An. fluviatilis James and An. annularis Van der Wulp are suspected to be vectors for malaria in Baluchistan province ${ }^{1}$.

Resistance to pyrethroids has been reported in major groups of insects including mosquitoes (i.e., Culex and Anopheles) from South America, Sudan, Sri Lanka, Nigeria, Burkina Faso, Egypt, Guatemala, USA, Turkey and Syria ${ }^{2-4}$. Many of the resistant insects serve as vectors of emerging diseases. Insecticide resistance is expected to directly affect the re-emergence of vector born diseases and it is expected to threaten disease control activities ${ }^{5-7}$. The resistance mechanisms involve esterases, glutathion S-transferases and/or monooxygenases $^{8}$. However, the most common mechanism of insecticide resistance in insects is modified level or activities of esterases that metabolize a wide range of insecticides ${ }^{9,10}$. Enhanced esterase activity has been detected in a number of insecticide resistant insects ${ }^{11,12}$. The esterase-based resistance mechanism has also been detected in An. stephensi $i^{13,14}$.

Interest has been generated in biochemical tests for detecting and monitoring insecticide resistance that are more specific than traditional bioassays. Detection of specific resistance mechanisms in individual insect and estimation of resistance genes in a population is the major objective of such tests $^{15}$. Most biochemical approaches developed thus far, aim at the detection or measurement of enzymatic reaction in homogenates of a single insect and the use of electrophoretic analysis, filter paper tests or micro titer plate assays. Many biochemical tests are concerned with the detection of organophosphate and carbamate resistance due to reduced sensitivity of acetylcholinesterase ${ }^{16-18}$ or increased detoxification by highly active esterases in mosquitoes ${ }^{19,20}$.

In Pakistan (Punjab), malathion (organophosphate) was used to control mosquitoes before 1991. However after 1991 malathion was replaced gradually by deltamethrin (synthetic pyrethroid), due to emergence of resistance in An. stephensi against malathion, as reported from some areas of Pakistan (Unpublished Surveillance data of communicable disease control programme of Punjab Health Department). Deltamethrin produced $100 \%$ mortality in An. culicifacies and An. stephensi and up to year 2002 no resistance has been reported against deltamethrin in country. The aim of present study was to 
investigate the following concerns:

(1) Are there any deltamethrin resisitant An. stephensi populations in the Lahore district?

(2) Is there any difference in the levels of esterases in different populations of An. stephensi?

(3) Is there any difference in the quantity of esterases in male and female specimens of a population?

(4) How many types of esterases are present in different populations of An. stephensi in Lahore district?

(5) Is there any correlation between levels of esterases and previous exposure of insecticides?

\section{EXPERIMENTAL}

Selection sites: On the basis of surveillance and operational data from the communicable disease control programme of the health department district Lahore. We have selected three localities (i.e., Jaman, Mohlanwal and Noorpur Bhatta A1) from rural areas for sampling. At present An. stephensi is found only in rural areas of the district. Moreover mosquito populations at these localities have a history of exposure to pyrethroids, especially deltamethrin used in the public health program ${ }^{1}$.

Sampling: During this study, An. stephensi were collected from living rooms, stores and animal sheds using an aspirator (WHO, 1992). Total sample size was 1200 (400 individual from each locality). Identification was made using the pictorial key developed by the National Institute of Malaria Research and Training, Lahore, a sub-center of the National Institute of Health, Islamabad.

Mosquitoes were divided into two groups. One group of female mosquitoes was subjected to adult bioassay tests and the second was stored at $-20^{\circ} \mathrm{C}$ for further biochemical estimation of esterases and polyacrylamide gel electrophoresis.

Adult bioassays: Blood-fed females $(n=25)$ and males $(\mathrm{n}=25)$ of An. stephensi were used for the bioassays ${ }^{21}$. Mosquitoes collected from each locality were exposed for $1 \mathrm{~h}$ to $5 \%$ deltamethrin impregnated papers using WHO standard exposure tubes ${ }^{3}$ and then transferred to holding tubes (tubes without impregnated papers). Three replicate tests were performed. Mortality of mosquitoes on control and treated papers was recorded after $24 \mathrm{~h}$ post exposure.

Biochemical estimation of esterases: Esterase activity was measured in homogenates of single adult mosquitoes of almost same size $(\mathrm{n}=25)$. Each adult was homogenized in 50 $\mu \mathrm{L}$ of $40 \mathrm{mM}$ phosphate buffer, $\mathrm{pH} 7.0$, containing $0.01 \%$ (w/v) of Triton $\mathrm{X}-100^{19}$. The homogenate was centrifuged at 13000 rpm for $5 \mathrm{~min}$. Supernatant was used in assay with $\alpha$-naphthyl acetate (substrate A) and $\beta$-naphthyl acetate (substrate B). The reaction mixture contained $30 \mu \mathrm{L}$ homogenate, $60 \mu \mathrm{L}$ substrate solution $(0.1 \mathrm{M}), 100 \mu \mathrm{L} \operatorname{SDS}(5 \%)$ and $1440 \mu \mathrm{L}$ phosphate buffer. After an incubation period of $0.5 \mathrm{~h}$ at $37{ }^{\circ} \mathrm{C}$, optical density was recorded at a wave length of $620 \mathrm{~nm}$ for $\alpha$-naphthyl acetate and at $545 \mathrm{~nm}$ for $\beta$-naphthyl acetate. The amount of esterase present was calculated by converting the optical density values into $\mathrm{ng} / \mathrm{mL}$ of esterase using standard curves, which were prepared with different concentrations of $\alpha$ - and $\beta$-naphthol (Fig. 1a and b).

Polyacrylamide Gel electrophoresis: Polyacrylamide gel electrophoresis was performed to separate different types of $\alpha$ - and $\beta$-esterases in different populations of An. stephensi. Only females of equal size were used for this purpose. Two concentrations of gel were used in polyacrylamide gel electrophoresis i.e., separating gel and staking gel. Clean gel plates were clamped firmly after placing spacers on the two sides of the gel plates. The plates were surrounded by gaskets on three sides to prevent any leakage. The separating gel (8\%) was prepared by mixing $9.7 \mathrm{~mL}$ distilled water, $5.3 \mathrm{~mL}$ acrylamide and bisacrylamide $(30 \%), 5 \mathrm{~mL}$ tris buffer $(\mathrm{pH}$ 8.8), $0.2 \mathrm{~mL}$ SDS (10\%), $0.2 \mathrm{~mL}$ ammonium persulphate $(10 \%)$ and $0.012 \mathrm{~mL}$ tetramethylethylenediamine. This solution was poured into the space between the two glass plates. The staking gel was prepared by mixing $3.4 \mathrm{~mL}$ distilled water, $830 \mu \mathrm{L}$ acrylamide and bisacrylamide $(30 \%), 630 \mu \mathrm{L}$ tris buffer (pH 6.8), $50 \mu \mathrm{L} \operatorname{SDS}(10 \%), 50 \mu \mathrm{L}$ ammonium per-sulphate $(10 \%)$ and $5 \mu \mathrm{L}$ tetramethyl ethylenediamine.
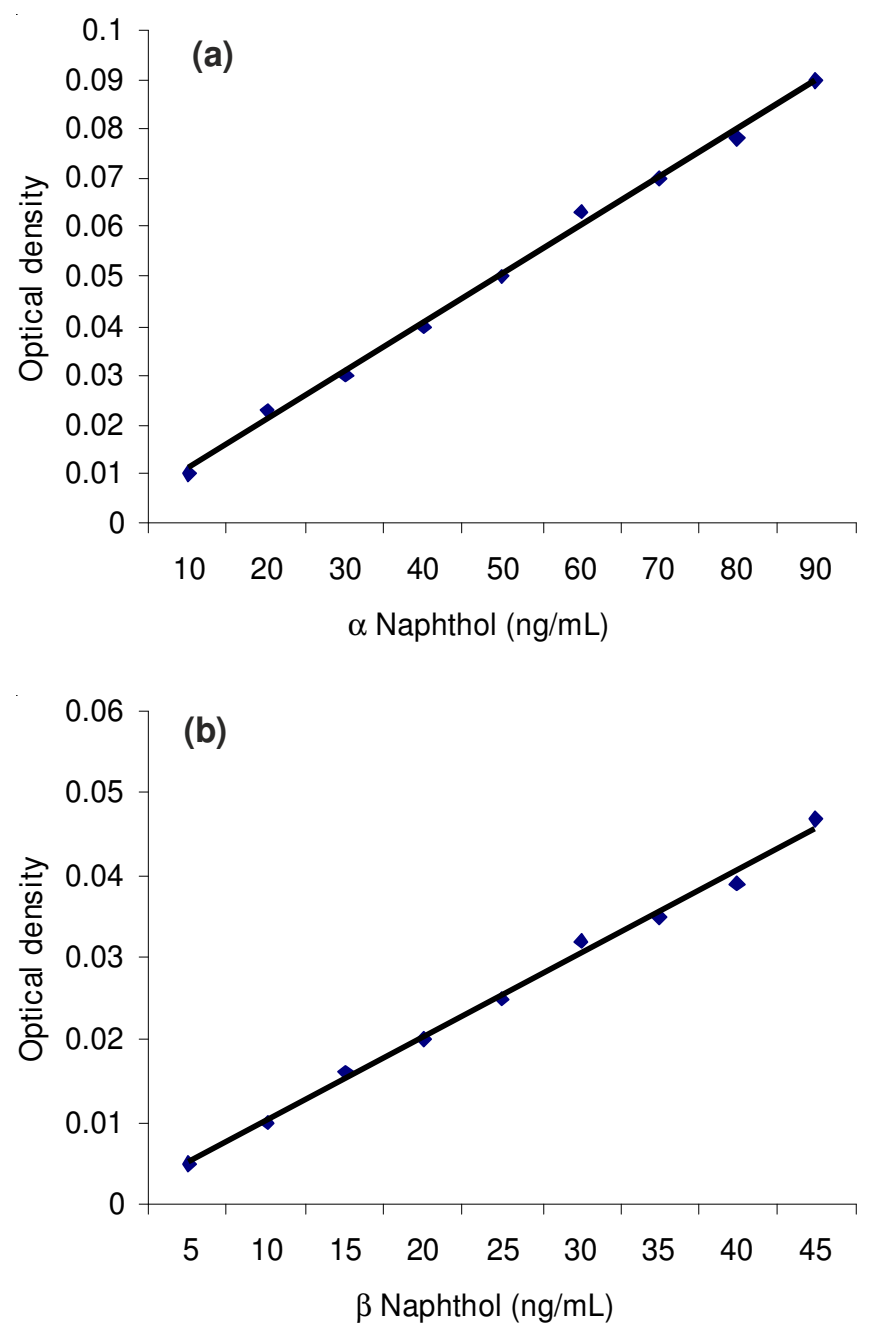

Fig. 1. Standard curve for $\alpha$-naphthol (a) and $\beta$-naphthol (b)

The staking gel was poured over the separating gel and a comb was inserted to make the wells. From each locality 15 female mosquitoes of same size were randomly selected and crushed in $300 \mu \mathrm{L}$ phosphate buffer. The homogenate was centrifuged at $13000 \mathrm{rpm}$ for $5 \mathrm{~min}$. From the supernatant $100 \mu \mathrm{L}$ of sample was mixed with $100 \mu \mathrm{L}$ of $\alpha$ - or $\beta$-naphthyl acetate and then incubated for $35 \mathrm{~min}$ at $37^{\circ} \mathrm{C}$. Then $150 \mu \mathrm{L}$ sample buffer was also added to the sample and heat shock 
was given to the sample for $5 \mathrm{~min}$. Gel plates were adjusted in a vertical position to the gel apparatus. An electrode buffer was added in both the upper and lower tanks of the gel apparatus. The gel was run at $150 \mathrm{v}$ with continuous cooling at $4{ }^{\circ} \mathrm{C}$ until the dye reached the lower plates. The gel was stained with fast blue RR stain for ten minutes. De-staining was done by methanol to remove the extra stain. When bands of esterases became clear, the gel pattern was studied.

Statistical analysis: Fisher's exact test was used to compare the mortality rate of mosquitoes at different time intervals. Paired $t$-test was used to compare the mortality in male and female mosquitoes.

Levels of esterases in different populations of $A n$. stephensi were compared by using ANOVA. Paired $t$-test was also used to compare the level of esterases in male and female mosquitoes using Minitab Software (version 13.3)

\section{RESULTS AND DISCUSSION}

Adult bioassays: Mosquitoes from all three populations were found susceptible using the WHO recommended dose of deltamethrin (i.e., $5 \%$ ). After 24 h exposure, $100 \%$ mortality was recorded. The mortality response of An. stephensi was highest between 3 to $12 \mathrm{~h}$ after insecticide exposure in all populations (Fig. 2). In all three populations mortality over time was significantly higher in males than females $(\mathrm{t}=4.99$; $\mathrm{P}<0.001)$.

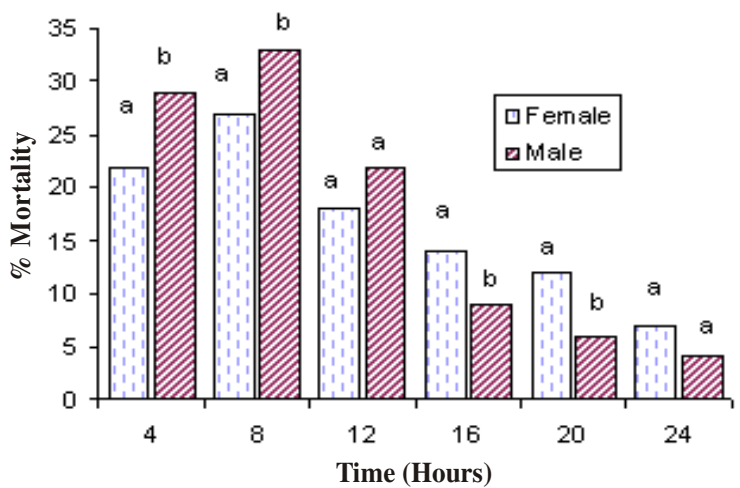

Fig. 2. Percent mortality of An. stephensi after $1 \mathrm{~h}$ exposure to deltamethrin (combined for three populations); Note: Bars in the figure with different superscripts are showing significant difference in percent mortality

Biochemical estimation of esterases: One-way ANOVA, performed to compare the levels of esterases in three different populations of An. stephensi, showed statistically significant variation $(\mathrm{F} 2,72=4.29 ; \mathrm{P}<0.05)$. The highest level of esterase activity was recorded in the Jaman population while the lowest was seen in the population from Noorpur Bhatta A1. It was observed that the mosquito populations from Jaman had high esterase activity against $\beta$-naphthyl acetate (Substrate B) while the other two populations (i.e., Mohlanwal and Noorpur Bhatta A1) had high esterase activity against $\alpha$-naphthyl acetate (Substrate A). Moreover, all three populations displayed a large variation in the levels of both estrases A and B. Females were found to have significantly higher activity of esterase $\mathrm{A}(\mathrm{t}=4.99$; $\mathrm{P}<0.001)$ and esterase $\mathrm{B}(\mathrm{t}=5.02 ; \mathrm{P}<0.001)$ than males.

Polyacrylamide gel electrophoresis: Results of polyacrylamide gel electrophoresis indicated differential profiles regarding

the mobility, intensity and number of bands for esterases A and esterases B. Only one band (A1) was observed for esterase A in all three populations (Fig. 3a). Differential band patterns were observed for esterases B in populations of three localities (Fig. 3b). Two bands, each of similar nature were observed in all the three populations. One slow moving band of high intensity occupied the upper part of the gel in the samples of all localities and was labeled as B1 while the narrow band (B3) of less intensity was present in the bottom of gels of all samples. In the population of Jaman a third bands (B2) of moderate intensity was present between B1 and B3. This band was absent in the population of Noorpur Bhatta A1 and Mohlanwal.

A
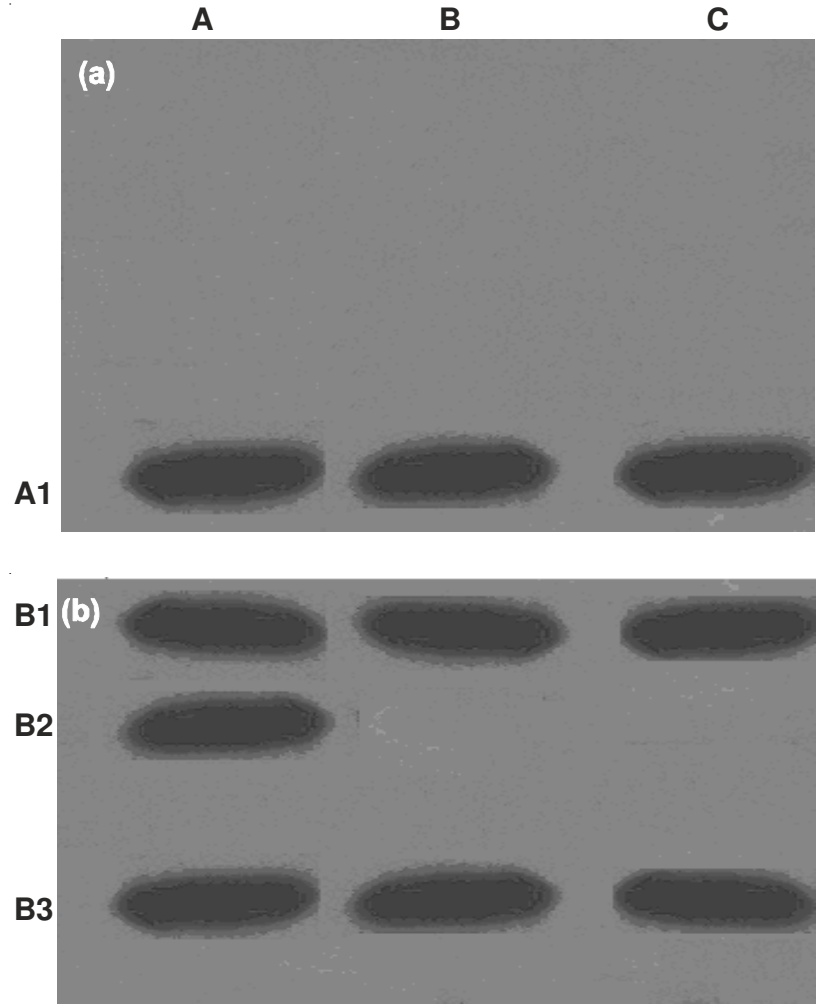

Fig. 3. Polyacrylamide gel of Anopheles stephensi showing esterase A (a) and esterases B; Note: A = Jaman, B = Mohlanwal, C = Noorpur Bhatta A1

Pyrethroid resistance is emerging despite early optimism that because of its rapid toxicological action this newest large class of insecticides would not produce resistance. Several studies have reported the development of resistance against pyrethroids in mosquitoes in different parts of the world such as Middle East, South America, Sudan, Sri Lanka, Nigeria, Burkina Faso, Egypt, Guatemala, USA, Turkey and Syria ${ }^{2,3}$. Results obtained during bioassays in the present study revealed that individuals of all three populations were susceptible to the WHO recommended dose $(5 \%)$ of deltamethrin. The mortality was higher between $3^{\text {rd }}$ and $12^{\text {th }}$ hour and no knockdown resistance was found. Present results were in contrast with the findings of Enayati et al. ${ }^{22}$ who reported knockdown resistance in An. stephensi from Dubai. Ganesh et al. ${ }^{23}$ also reported tolerance to deltamethrin in larvae of An. stephensi from Mysore. Though a small proportion of the population from Jaman and Mohlanwal had increased level of esterase A and 
esterase B, the populations were susceptible during bioassays. Ocampo and Wesson ${ }^{24}$ also reported that a small proportion of An. pseudopuntipennis from Colombia, having high levels of esterases were susceptible during bioassay against pyrethroids.

Significant variation was recorded in esterase levels among populations and within the tested populations. Pasteur et al..$^{15}$ also observed variation in quantity of esterases in fieldcollected mosquitoes. The population from Jaman showed more variation in enzyme activity to hydrolyze the $\beta$-naphthyl acetate (Substrate B) compared to populations at Mohlanwal and Noorpur Bhatta A1. Variations of esterases in these three populations may be due to exposure of individuals to organophospate compounds. All localities have agricultural fields and organophosphates are being used for agriculture in these areas. Greater variation in quantity of esterases B in Jaman than the other two localities may be related to the fact that the area used for agriculture in this locality is much greater than the other two localities. Thus the use of organophospate compounds is much heavier in this locality.

According to Roush ${ }^{25}$ esterases are important in resistance to organophosphate insecticides in mosquitoes and occasionally they play role against pyrethroids. $\alpha$-Naphthyl acetate showed high activity with mosquito homogenates of Noorpur Bhatta A1 and Mohlanwal, indicating the predominance of esterases $\mathrm{A}$ in these populations. The increased levels of esterase $\mathrm{A}$ have no contribution toward the development of resistance against deltamethrin in these populations of An. stephensi as demonstrated by the lack of resistance detected in WHO bioassays. Organophosphate compounds are still in use for agriculture practice might be the reason of increased level of esterases A. However, the mosquito homogenates of Jaman populations hydrolyzed the beta naphthyl acetate faster than $\alpha$-naphthyl acetate which is an indication for the dominance of esterases B. Ganesh et al. ${ }^{23}$ have found significant correlation between increased esterases B and development of tolerance against deltamethrin in the larvae of An. stephensi. However, in Jaman esterase B may be related to greater exposure of individuals to the organophospate because population was also found susceptible to deltamethrin during bioassays. Mouches et al. ${ }^{26}$ and Beyssat et al. ${ }^{27}$ have also reported that increased esterases $\mathrm{B}$ are responsible for organophospate compounds resistance in An. stephensi and Cx. quinquefasciatus. Tahir et al. ${ }^{28}$ has also reported an increase in esterases B and esterases A in different populations of $C x$. quinquefadciatus from the district of Lahore not related with pyretheroid resistance. Results of the present study are also in contrast with the result of Bull et al. ${ }^{29}$ who found greater esterases B in pyrethroid-resistant populations. This could be interpreted to mean that this esterase is involved in pyrethroid resistance but according to present study esterase A is not involved in pyrethroid resistance because those populations, which have greater exposure to pyrethroid showed high values of optical densities with $\alpha$ naphthyl acetate.

Higher levels of esterases A and esterases B were found in female An. stephensi than males in the present study. This difference level in males and females might be associated with the longevity of females compared to males. During their life span, females have greater exposure to insecticides, which may result in enhanced level of detoxifying enzyme in comparison to males. These results are contrary to Krafsur and Ernst ${ }^{30}$ who reported high levels of only esterases $B$ in female but not A in horn flies. Some studies have shown that both esterases A and $\mathrm{B}$ are high in females ${ }^{19,20}$.

During the present study differential profiles for esterases A and esterases B regarding mobility, intensity and number of bands was noted. A differential profile of esterases A and esterases B has also been reported by Ganesh et al. ${ }^{23}$ in An. stephensi and An. culcifacies. Only one kind of band was observed in all three sampled populations for esterases A. Dubash et $a l .{ }^{31}$ has reported four electrophoretic variants of esterases A in laboratory strain of An. culicifacies. Bisset et al. ${ }^{32}$ reported two types of esterasess $\mathrm{A}$ in $C x$. quinquefasciatus from Cuba and Tahir et al. ${ }^{28}$ has reported one type of esterases $\mathrm{A}$ in $C x$. quinquefasciatus from Lahore Pakistan.

For esterases B, three bands (B1, B2 and B3) in Jaman and two bands in Noorpur Bhatta A1 and Mohlanwal populations were observed. A third band (B2) was present only in Jaman and was absent in Noorpur Bhatta A1 and Mohlanwal. Beyssat et al. ${ }^{27}$ has reported one band of esterases B in An. stephensi, Bisset $^{33}$ has reported three types of esterases B in $C x$. quinquefasciatus from Cuba whereas Tahir et al. ${ }^{28}$ has reported three types of esterases B from Lahore, Pakistan.

From the present studies it is concluded that present variations and high level of esterases in populations of $A n$. stephensi in district Lahore are not producing resistance against deltamethrin. Males have lower levels of esterases compared with females. There is only one type of esterase $\mathrm{A}$ in all populations while 2-3 types of esterases B are present.

\section{REFERENCES}

1. TAMA and DOMC Technical Assistance Management Authority and Directorate of Malaria Control, Malaria Microscopy Training Manual Skill Level-1 (2007).

2. C.A. Malcolm, Parasitol. Today, 4, S13-S15 (1988).

3. WHO, Vector resistance to pesticides. 15th report of the Expert Committee on Vector Biology and Control, Tech. Rep. Ser., p. 818 (1992).

4. X. Li, M.A. Schuler and M.R. Berenbaum, Ann. Rev. Entomol., 52, 231 (2007).

5. M.R. Ghosh, Concept of Insect Control, New Age International Publishers, New Delhi, pp. 97-98 (1989).

6. D.J. Krogstad, Epidemiol. Rev., 18, 77 (1996)

7. D. Yewhalaw, W. Vanbortel, L. Denis, M.L. Coosemans, L. Duchateau and N. Speybroeck, Am. J. Trop. Med. Hyg., 83, 122 (2010).

8. J.G. Scott, N. Liu and Z.M. Wen, Comp. Biochem. Physiol., 121, 147 (1998).

9. F.J. Oppenoorth, Pestic. Biochem. Physiol., 22, 187 (1984).

10. M.D.B. Perera, J. Hemingway and S.H.P.P. Karunaratne, Malar. J., 7, 168 (2008).

11. J. Hemingway and H. Ranson, Ann. Rev. Entomol., 45, 369 (2000).

12. J.C. Hsu, W.J. Wu and H.T. Feng, Plant Prot. Bull., 46, 255 (2004).

13. J. Hemingway and S.H.P.P. Karunaratne, Med. Vet. Entomol., 12, 1 (1998).

14. M. Raymond, A. Callaghan, P. Fort and N. Pasteur, Nature, 350, 151 (1991).

15. N. Pasteur, A. Iseki and G.P. Georghiou, Biochem. Genet., 19, 909 (1981).

16. M. Raymond, N. Pasteur, D. Fournier, A. Cuany, J. Berg and M. Magnin, Comptes Rendus de l'Academie des Science, Series III, p. 509 (1985).

17. J. Hemingway, I.G.K. Jayawardena, I. Weerasinhe and J.R.P. Herath, Bull. Entomol. Res., 77, 57 (1987).

18. W.G. Brogdon, J.H. Hobbs, St. Y. Jean, J.R. Jacques and B.L. Charles J. Am. Mosq. Control. Assoc., 4, 152 (1988).

19. G.P. Geoghiou and N. Pasteur, J. Econ. Entomol., 71, 201 (1978).

20. A.T. Rees, N.W. Field and A.M.J. Hitchen, J.Am. Mosq. Control. Assoc., 1, 23 (1985). 
21. WHO, Prevention, Diagnosis and Treatment of Insecticide Poisoning. WHO/VBC/84. 889 (1981).

22. A.A. Enayati, H. Vatandoost, H. Lasonni, H. Townson and J. Hemingway, Med. Vet. Entomol., 17, 138 (2003).

23. K.N. Ganesh, J. Urmila and V.A. Vijayan, Indian J. Med. Res., 117, 30 (2003)

24. C.B. Ocampo and D.M. Wesson, Am. J. Trop. Med. Hyg., 71, 506 (2004).

25. R.T. Roush, Parasitol. Today, 9, 174 (1993)

26. C. Mouches, M. Magnin, J.B. Berge, M. De. Silvestri, V. Beyssat, N. Pasteur and G.P. Georghiou, Proc. Natl. Acad. Sci., USA, 84, 2113 (1987).
27. V. Beysatt-Arnaouty, C. Mouches, G.P. Geoghiou and N. Pasteur, J. Am. Mosq. Contr. Assoc., 5, 196 (1989).

28. H.M. Tahir, A. Butt and S.Y. Khan, J. Parasitol. Vector Biol., 1, 19 (2009).

29. D.L. Bull, L.R. Harris and W.N. Pryor, J. Econ. Entomol., 81, 449 (1988).

30. E.S. Krafsur and C.M. Ernst, J. Med. Entomol., 23, 188 (1986).

31. C.J. Dubash, K.S. Richard and H.B. Richard, J. Heredity., 73, 209 (1982).

32. J.A. Bisset, M.M. Rodríguez, C. Díaz, E. Ortiz and M.C. Marquetti, Bull. Entomol. Res., 80, 160 (1990).

33. J.A. Bisset, Med. Vet. Entomol., 5, 223 (1991). 